\title{
Geometric point-circle pentagonal geometries from Moore graphs
}

\author{
Klara Stokes * \\ School of Engineering Science, University of Skövde, 54128 Skövde Sweden \\ Milagros Izquierdo \\ Department of Mathematics, Linköping University, 58183 Linköping Sweden
}

Received 27 December 2014, accepted 13 October 2015, published online 15 November 2015

\begin{abstract}
We construct isometric point-circle configurations on surfaces from uniform maps. This gives one geometric realisation in terms of points and circles of the Desargues configuration in the real projective plane, and three distinct geometric realisations of the pentagonal geometry with seven points on each line and seven lines through each point on three distinct dianalytic surfaces of genus 57 . We also give a geometric realisation of the latter pentagonal geometry in terms of points and hyperspheres in 24 dimensional Euclidean space. From these, we also obtain geometric realisations in terms of points and circles (or hyperspheres) of pentagonal geometries with $k$ circles (hyperspheres) through each point and $k-1$ points on each circle (hypersphere).
\end{abstract}

Keywords: Uniform map, equivelar map, dessin d'enfants, configuration of points and circles

Math. Subj. Class.: 05B30, 05B45, 14H57, 14N20, 30F10, 30F50, 51E26

\section{Introduction}

A compact Klein surface $S$ is a surface (possibly with boundary and non-orientable) endowed with a dianalytic structure, that is, the transition maps are holomorphic or antiholomorphic (the conjugation $z \rightarrow \bar{z}$ is allowed). If the surface $S$ admits analytic structure and is closed, then the surface is a Riemann surface. By the uniformization theorem each Klein surface is a quotient $S=U / G$, where $U$ is either the Riemann sphere, the complex Euclidean plane or the hyperbolic plane, and $G$ is a group without elliptic elements. In the case of surfaces without boundary the group $G$ is torsion-free.

* Partially supported by the Spanish MEC project ICWT (TIN2012-32757) and ARES (CONSOLIDER INGENIO 2010 CSD2007-00004).

E-mail addresses: klara.stokes@his.se (Klara Stokes), milagros.izquierdo@liu.se (Milagros Izquierdo) 
The surface inherits the geometry of its universal covering space $U$ through this quotient. Incidences between lines and circles in $S$ follow the same axioms as in the covering space and geodesics on the surface come from lines in the covering space. In what follows, terms like line and circle will refer to such geometric objects, if not defined otherwise.

A map is a drawing of a graph on a surface such that the complement of the drawing is a disjoint union of topological discs called faces. So a map consists of a set of vertices, a set of edges and a set of faces. The genus of a map is the genus of the surface in which the graph is embedded, and can be calculated through the Euler characteristic using a generalization of Euler's polyhedron formula. Given a map with $|V|$ points, $|E|$ edges and $|F|$ faces, the Euler characteristic is $\chi=|V|-|E|+|F|$. The genus $g$ of an orientable surface satisfies $\chi=2-2 g$ and the genus $h$ of a non-orientable surface satisfies $\chi=2-h$. By considering the map as the lifting of the segment $[0,1]$ in $\mathbb{C}$, the map determines the structure of the dianalytic surface. In general, a given surface allows different maps, and a given graph can be embedded as a map on different surfaces [18, 5, 4]. However, among the different maps of a graph there is one which has the largest Euler characteristic, then called the Euler characteristic of the graph. This map will have the smallest orientable or non-orientable genus of all maps of this graph, depending on whether it is orientable or not.

It makes sense to consider both the smallest orientable and the smallest non-orientable genus of the graph. For example, the orientable genus of a planar graph is the genus of the sphere, which is 0 . This is also the orientable genus of the 1-skeleta of the Platonic surfaces. The Petersen graph is not planar and so it has orientable genus at least 1 . Since it can be drawn without crossings on the torus, it has orientable genus exactly 1 . The hemidodecahedron is the abstract polyhedron obtained by identifying antipodal points in the dodecahedron. The 1-skeleton of this polyhedron is the Petersen graph, defining a map of the Petersen graph in the real projective plane, so the non-orientable genus of the Petersen graph is 1 .

The study of configurations in projective real or complex plane is a classical subject in geometry. Configurations appear naturally as arrangements of lines, planes or circles in a geometric plane or space. In contrast with the situation when graphs are realised as maps on surfaces, the requirement that there should be no crossings on the surface other than the incidences defined by the configuration is typically relaxed (although not always). For example, Hilbert and Cohn-Vossen [16] define a planar point-line configuration as follows.

"A plane configuration is a system of $v$ points and b straight lines arranged in a plane in such a way that every point is incident with $r$ lines and every line is incident with $k$ points."

Note that it is not required that the $b$ lines should meet only in the $v$ points, only the incidences in the distinguished points are important. However, extra incidences on these points are often regarded as an anomaly.

For example, consider Desargues' Theorem, which is a theorem regarding the realisation of the configuration in Figure 1 in projective planes. In a projective plane every pair of lines intersect, therefore every pair of the 10 lines in the configuration in Figure 1 must meet at some point. Some of these points do not belong to the configuration. Similarly, there is of course a line between each pair of points, but some of these lines do not belong to the configuration. What makes it a configuration is the fact that in any of the 10 points there are $r=3$ of the 10 lines intersecting, and on any of the 10 lines there are $k=3$ of the 10 points. However, if it was drawn so that a 4 th of the 10 points accidently were on an 


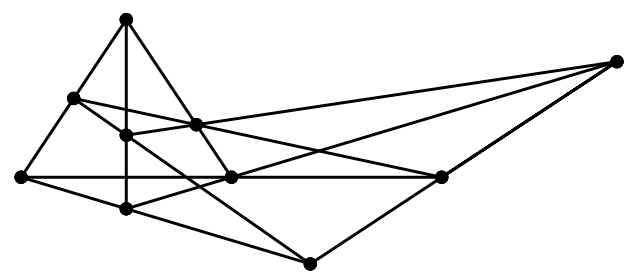

Figure 1: Desargues' Theorem: two triangles are perspective from a point if and only if they are perspective from a line.

extra line, then some lines would have 3 points and others would have 4 points, making the configuration degenerate. An $(r, k)$-combinatorial configuration is a set of incidences between two sets of $v$ and $b$ elements called points and lines respectively, defined in analogy with the planar and linear definition above, but without considering realisability in some geometric space; see for example [13,22]. A combinatorial configuration is called linear if each pair of lines meet at most once. Linear combinatorial configurations are often simply called combinatorial configurations. Combinatorial configuration with $k=2$ or $r=2$ are graphs or their duals are graphs, respectively. Therefore it is typically required that $r \geq 3$ and $k \geq 3$.

A pentagonal geometry is a (linear) combinatorial configuration in which, for any point $p$, all points that are not collinear with $p$ are on a single line, which is called the opposite line of $p$. A pentagonal geometry has $\operatorname{order}(k, r)$ if there are $r$ lines through each point and $k$ points on each line [1]. There are two classes of lines in a pentagonal geometry, lines that are the opposite line of some point, and lines that are not. A pentagonal geometry with no non-opposite lines is self-polar by the polarity that associates each point with its opposite line. The deficiency graph $(P, E)$ of a configuration is a graph with vertex set $P$, consisting of the points of the configuration, and edge set $E$, consisting of all the pairs $(p, q)$ such that the points $p, q \in P$ are not collinear. In a pentagonal geometry, for each $p \in P$ the opposite line of $p$ is formed by the points that are neighbours to $p$ in the deficiency graph. When $r=k$, the number of points equals the number of lines, so there are no non-opposite lines, and all lines are defined by the neighbourhood of some point in the deficiency graph. Given the deficiency graph it is then possible to construct the pentagonal geometry by drawing a (combinatorial) line through the neighbourhood of each point in the deficiency graph. This construction of pentagonal geometries was described in [1], where it also was proved that pentagonal geometries with $r=k$ are exactly the ones with a Moore graph (of diameter two) as deficiency graph.

There are only three known Moore graphs of diameter 2: the cycle graph of length 5, the Petersen graph, and the Hoffman-Singleton graph. These graphs have valency 2, 3 and 7, respectively. They are unique for their valencies [17]. The existence of a Moore graph of valency 57 is still an open question. The pentagonal geometries obtained from these graphs are, respectively, the ordinary pentagon, the Desargues configuration (Figure 1) and a pentagonal geometry with parameters $(7,7)$ and with 50 points and 50 lines. In [1], it was also proved that all pentagonal configurations of order $(k, k+1)$ can be constructed from pentagonal geometries of order $(k+1, k+1)$ through the removal of one point and its opposite line. There are therefore at most three such pentagonal geometries, with $k=2,6$ 
and maybe 56 .

The construction of pentagonal geometries from a graph with a combinatorial line through the neighbourhood of each vertex can also be used to construct other configurations. Indeed, the same construction works for any graph with the property that any two vertices have at most one common neighbour. In other words, the graph should be without cycles of length 4. This construction seems to appear first in an article by Lefèvre-Percsy, Percsy and Leemans, as the neighbourhood geometry (of rank 2) of a graph [20], and later, in the context of geometric realisations of configurations in articles by Gévay and Pisanski $[9,10]$. If the graph can be drawn in the real Euclidean plane in such a way that a circle can be traced through the neighbours of each point, then the drawing and the construction together give rise to a geometric point-circle configuration in the real Euclidean plane [10]. For example, any 3-regular graph has this property, defining a point-circle $(3,3)$-configuration in the real Euclidean plane. Also unit-distance graphs can be used for the same purpose. Indeed, a circle is defined as the collection of points at a given distance from the center of the circle. As an extra feature, a unit-distance graph gives an isometric point-circle configuration, in which all circles have the same radius.

As was observed in [9], a point-plane configuration in real Euclidean 3-space, constructed through a similar construction from the 1-skeleton of a 3-polytope, defines a pointcircle configuration in the real Euclidean plane through stereographic projection whenever the points in each plane are concyclic. In particular, it was proved in [9] that any Platonic or Archimedean solid gives a point-circle configuration on the Riemann sphere, and that the circle-preserving property of the stereographic projection implies that any point-circle configuration drawn on the sphere can also be drawn in the real Euclidean plane.

In this article we will generalize this construction on the sphere to surfaces in general. This construction is motivated by the study of geometric realisations of pentagonal geometries.

\section{Constructing configurations of points and isometric circles on sur- faces}

The geometric construction of Gévay and Pisanski described above does not require an embedding without crossings of the graph. Rather, what the construction requires from the graph embedding is that the neighbours of each vertex are concyclic [10]. On the sphere, any circle is a planar section, so any point-circle configuration gives a point-plane configuration in 3-space. Since more than 3 points in a plane are not necessarily concyclic, the converse is not true in general when $k>3$.

A nice way of making neighbours concyclic is to mimic the idea of using a map of the 1 -skeleton of a convex polytope. A regular tiling $(p, q)$ of the universal covering space $U$ of a Riemann surface is a collection of congruent polygons which partitions and fills up the entire space, in such a way that $p q$-gons meet at each vertex. The stabilizer of this tiling is a subgroup of a triangle group $\Gamma(p, 2, q)$. Since the polygons are congruent, the neighbours of each vertex are concyclic on isometric circles. The distance is the spherical, the Euclidean or the hyperbolic distance respectively.

Definition 2.1. A uniform map of type $(p, q)$ on a Riemann surface with universal covering space $U$ is the quotient of a regular tiling of $U$ of type $(p, q)$ by the action of a torsion-free group $G \subseteq \Gamma(p, 2, q)$.

This terminology comes from the theory of dessin d'enfants $[12,18,26]$, where also 
the term uniform dessin d'enfants is used. In the theory of tilings and polytopes the word uniform map instead refers to a map with an automorphism group acting transitively on the vertices. In the literature of tilings and polytopes, our uniform maps are instead known as equivelar maps. In particular, our uniform maps are not necessarily vertex transitive.

In a uniform map of type $(p, q)$ the vertices have valency $p$, the edges have valency 2 , and the faces have valency $q$. Any map with this property is a uniform map of type $(p, q)$. A map is regular if its automorphism group acts transitively on triples of incident vertices, edges and faces, that is, on the flags. This implies that a regular map is always uniform.

Isometric circles through the neighbours of each vertex of a regular tiling of $U$ will be mapped to isometric circles through the neighbours of each vertex of the corresponding uniform map on $U / G$. Since each circle contains $p$ points and $p$ circles goes through each point, this construction gives a configuration of points and circles on the surface, and we have proved the following.

Theorem 2.2. A uniform map on a surface produces a configuration of points and isometric circles on the same surface.

On the sphere, this construction gives a configuration of points and isometric circles which can be taken to a configuration of points and non-isometric circles on the Euclidean plane through stereographic projection from a suitable point.

The uniform maps on the sphere are regular. Consequently, there are two infinite families of uniform maps of the sphere, the hosohedra of type $(n, 2)$ for $n \geq 1$, consisting of $n$ digons meeting at two antipodal vertices, and the dihedra of type $(2, n)$ for $n \geq 1$, consisting of two $n$-gons meeting at $n$ vertices along a meridian. The result from applying Theorem 2.2 to a hosohedron is a degenerate configuration consisting of two points and two circles of radius zero, each point occuring with multiplicity $n$ on one of the circles. By instead using a dihedron one obtains a configuration of $n$ points and $n$ circles with two points on each circle. This configuration is connected if $n$ is odd, otherwise the configuration consist of two disconnected components.

A part from the two infinite families just described, which result in configurations of limited interest, there are only five more uniform maps on the sphere, corresponding to the Platonic solids. Of the resulting configurations, there is only one which is linear when regarded as a combinatorial configuration.

Theorem 2.3. The only linear point-circle configuration (with $r>2$ and $k>2$ ) coming from a uniform map on the Riemann sphere is the $\left(20_{3}, 20_{3}\right)$-configuration on Figures 1 , 2, 3 in [10], obtained from the dodecahedron projected on the sphere. In the real projective plane, the only linear point-circle configuration coming from a uniform map is the Desargues configuration, obtained from the hemidodecahedron.

Proof. The uniform maps of type $(p, q)$ on the sphere satisfying $p>2$ and $q>2$ are the Platonic solids. Gévay and Pisanski constructed point-plane configurations from all Platonic (and Archimedean) solids except the octahedron in [10]. The octahedron has the property that the planes through the neighbours of two antipodal vertices coincide. They also proved that their construction gives a combinatorial point-line configuration (i.e. in which any two combinatorial lines share at most one point) only if the graph does not have cycles of length 4 . The only Platonic solid graph without cycles of length 4 is the dodecahedron graph. The uniform maps in the real projective plane are obtained from the uniform maps on the sphere by identifying antipodal points. As we pointed out in the introduction, the hemi-dodecahedron is obtained from the dodecahedron in this way. 
The sphere has finite area, implying that each uniform tiling has a finite number of tiles. Hence the automorphism group of the tiling is finite and has a finite number of subgroups. Therefore the finite number of regular tilings with $p, q \geq 3$ of the sphere gives a finite number of uniform maps. The situation is different in the Euclidean and the hyperbolic plane. The Euclidean plane has a finite number of regular tilings (of types $(6,3),(3,6)$ and $(4,4))$, but here the area is infinite, resulting in infinitely many uniform maps. The hyperbolic plane has infinite area and there are infinitely many regular tilings, and consequently infinitely many uniform maps.

In comparison with the situation in the Euclidean plane, where it is possible to construct isometric point-circle configurations without starting with a planar unit-distance embedding of the graph, it is clear that in general it is not necessary to require the graph to be embedded as a uniform map on the surface. Isometric point-circle configurations can in some cases be obtained using other embeddings (non-uniform, non-congruent, with crossings) of the graph on the surface. However, in this article we focus on point-circle configurations coming from uniform maps, more precisely, on those coming from uniform pentagonal maps of Moore graphs of diameter 2.

\section{Geometric pentagonal geometries}

Here (in three subsections) we discuss different geometric realisations of pentagonal geometries, with focus on embeddings in Riemann surfaces.

\subsection{The ordinary pentagon}

The ordinary pentagon is the smallest non-degenerate pentagonal geometry. Its deficiency graph is the cycle graph on 5 vertices. This graph can also be seen as a point-line realisation of the configuration itself. The ordinary pentagon can also be constructed as a point-circle configuration with two points on each circle from its deficiency graph using the geometric construction by Gévay and Pisanski. So it can be argued that any point-line realisation of the ordinary pentagon produces a point-circle realisation of the same.

The cycle graph on 5 vertices has diameter 2 and girth 5, as do all Moore graphs (of diameter 2). The smallest number of edges in any face of an embedding of this graph on a surface is therefore 5. For example, it can be embedded in the Riemann sphere as a pentagonal cycle along one of the geodesics. This map has 5 vertices, 5 edges and 2 faces and so the orientable genus is 0 . We call it a pentagonal map, meaning simply that all faces have 5 vertices. By introducing one new vertex on the midpoint of each edge of this pentagonal spherical map, and identifying antipodal points in the resulting decagonal map one obtains a non-orientable pentagonal map with 1 face in the real projective plane, so the non-orientable genus is 1 . So the ordinary pentagon can be realised as a configuration of points and circles on the Riemann sphere (and consequently in the Euclidean plane), and in the real projective plane.

\subsection{The Desargues configuration}

The Desargues configuration is a $(3,3)$-configuration on 10 points and 10 lines. It is the pentagonal geometry with the Petersen graph, the 3-regular Moore graph, as deficiency graph. The polarity of the Desargues configuration is known as the von Staudt polarity [28](cf. [8]). Figure 1 shows a classical drawing of Desargues configuration in the 
real plane as the 10 points and 10 lines of Desargues' Theorem. There are plenty of geometric realisations of the Desargues configuration in terms of incidences of points and lines. Indeed, finite projective planes over finite fields are called Desarguesian since they admit the Desargues configuration as points and lines.

The automorphism group of the (combinatorial) Desargues configuration is $S_{5}$, the symmetric group acting on a set of five elements. When a configuration is realised geometrically, the automorphism group of the realisation is a subgroup of the automorphism group of the combinatorial configuration. Geometric realisations of the Desargues configuration were studied by Coxeter in [8], where he showed how to realise subgroups of $S_{5}$ as collineations of certain embeddings of the Desargues configuration in some geometric space. Among his collection of geometric realisations of the Desargues configuration, there are two which have the full automorphism group $S_{5}$. The first is due to Edge, who proved that in $P G(2,5)$, the interior points of a conic, together with the lines that are neither tangents nor secants to the same conic, form a Desargues configuration. The second is an embedding of the Desargues configuration on a non-orientable surface of Euler characteristic -5 . This embedding arises from a regular map of the Menger graph (collinearity graph) of the configuration on the surface, with automorphism group $S_{5}$. Coxeter observed that the 30 edges in this regular map are situated on 10 geodesics of the surface in such a way that the vertices of the map together with the 10 geodesics form a Desargues configuration of points and lines on the surface, which also has automorphism group $S_{5}$.

We saw in the introduction that any $(3,3)$-configuration can be realised as a configuration of points and circles in the Euclidean plane using Gévay and Pisanski's geometric spherical construction and an embedding of some 3-regular graph [10]. In particular this is true for the Desargues configuration, using an embedding of the Petersen graph. Gévay and Pisanski also showed how to make the circles isometric. Unit-distance embeddings of the graph always produce isometric circles, but some embeddings with edges of different lengths also work. They provided two examples of the Desargues configuration as a configuration of points and isometric circles in the real Euclidean plane, coming from a unit-distance and a non-unit-distance embedding of the Petersen graph, respectively. The automorphism group of these realisations are the cyclic group $C_{5}$ and the dihedral group $D_{5}[10]$.

We show now that the Desargues configuration also can be drawn as a point-circle configuration in the real projective plane from a pentagonal map of the Petersen graph. Indeed, the (Riemann) spherical $(3,3)$-configuration on 20 points and 20 circles constructed from the dodecahedron in [10] is the double cover of a $(3,3)$-configuration on 10 points and 10 circles in the real projective plane which can be constructed analogously from the hemidodecahedron. Since the 1-skeleton of the hemi-dodecahedron is the Petersen graph, it is easy to see that this configuration on 10 points and 10 circles is a point-circle realisation of the Desargues configuration. Figure 2 shows this point-circle configuration constructed in this way from the Petersen graph embedded as the 1-skeleton of the hemi-dodecahedron. Be aware that incidences outside the vertices may be accidental. The automorphism group of this realisation is the symmetric group $S_{5}$.

\subsection{The pentagonal geometry with the Hoffman-Singleton graph as deficiency graph}

The third and last pentagonal geometry that we will discuss in this article is the $(7,7)$ pentagonal geometry which has the Hoffman-Singleton graph as deficiency graph. The Hoffman-Singleton graph was first constructed by Hoffman and Singleton in 1960 [17]. It 


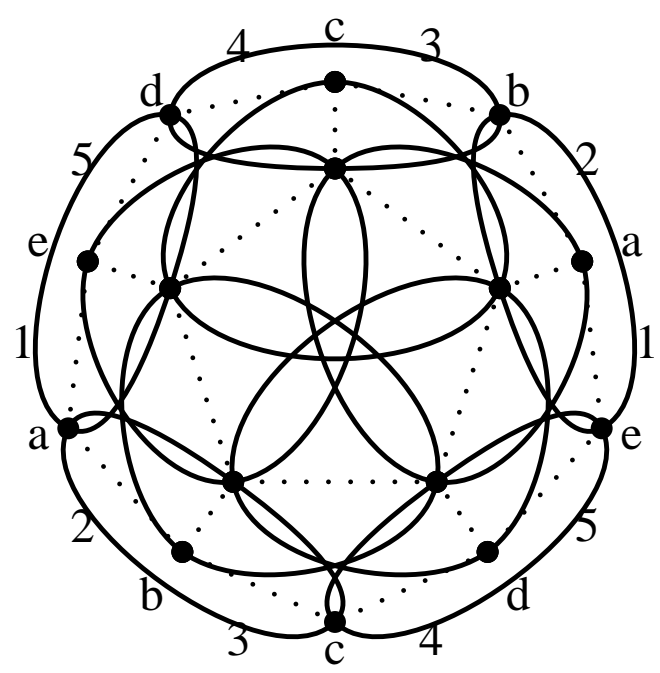

Figure 2: The Desargues configuration (black lines) obtained from the Petersen graph embedded in the real projective plane as the hemi-dodecahedron (dotted lines). Points are identified according to letters, and edges are identified according to numbers.

is a symmetric graph with automorphism group $P \Sigma U(3,5)=P S U(3,5) \rtimes C_{2}$, which has order 252000 .

The group $P S U(3,5)$ is the automorphism group of the Hermitian curve over $\mathbb{F}_{25}$. The first geometric construction of the Hoffman-Singleton graph in this curve was described by Benson and Losey [2] in 1971. Recently Shimada presented a unified construction of the Hoffman-Singleton graph, the Higman-Sims graph and the McLaughlin graph in this curve [24].

In a classical construction by Robertson [23](cf. [15]) the Hoffman-Singleton graph is obtained after connecting the vertices of 5 pentagons and 5 pentagrams. Later this construction was interpreted in terms of affine geometry over $\mathbb{F}_{5}$ by Hafner [15].

There is also the following construction of the Hoffman-Singleton graph due to Haemers [14]. Take as vertices the union of the points $v_{p}$ and the lines $v_{l}$ of $P G(3,2)$. Put an edge between a point vertex $v_{p}$ and a line vertex $v_{l}$ if $p$ is a point on $l$. This gives each point vertex valency 7 and each line vertex valency 3 . Also put an edge between two line vertices $v_{l}$ and $v_{l^{\prime}}$ if $l \cap l^{\prime}=\emptyset$. This makes the graph 7-regular. To see that this is the Hoffman-Singleton graph, observe that the girth is 5 and that there are 50 vertices.

Other geometric constructions of the Hoffman-Singleton graph are described for example in [3].

In all the constructions described above, except in the first two ([2, 24]), it is required that the vertex set be partitioned into two parts, and then the vertices in the different parts are represented by geometric objects of different types. We argue that in these cases what is dealt with are not geometric realisations of the Hoffman-Singleton graph, but geometric constructions. 
Our interest in this article is focused on geometric realisations of the $(7,7)$ pentagonal geometry in the classical sense. That means realisations of the configuration in terms of points and lines, or points and circles, in the plane or on some other two-dimensional surface. We are also interested in higher dimensional generalizations, hyperplanes instead of lines and hyperspheres instead of circles. In particular, all geometric realisations of the $(7,7)$ pentagonal geometry will be circular or spherical.

The construction of the $(7,7)$-pentagonal geometry from the Hoffman-Singleton graph associates the points of the pentagonal geometry with the vertices of the graph. Therefore we are interested in geometric realisations of the Hoffman-Singleton graph in which all the vertices are represented by geometric objects of the same type.

\subsubsection{The (7,7) pentagonal geometry as a point-circle configuration on a surface of characteristic $\mathbf{- 5 5}$}

Just as for the smaller Moore graphs of diameter 2, in a drawing without crossings of the Hoffman-Singleton graph on some surface, all faces will have at least 5 vertices. It can be seen from Eulers polyhedron formula that a map with only pentagonal faces will have the smallest possible genus. Indeed, since the Hoffman-Singleton graph has 50 vertices and 175 edges, the Euler characteristic of the map is $\chi=|V|-|E|+|F|=-125+|F|$, where $|F|$ is at most $350 / 5=70$, so $\chi$ reaches its largest value of -55 if all faces are pentagons. In that case the surface has non-orientable genus 57. It can be proved that such a map does exist, but cannot be a regular map [6]. Consequently, the automorphism group of the map will not be the full automorphism group of the graph. More precisely, there exist maps representing the Hoffman-Singleton graph which have as automorphism group the cyclic groups $C_{7}, C_{5}$ and the trivial group. These maps sit on non-orientable surfaces of the form $S=\mathbb{H} / G$, where $G$ is a torsion-free non-normal subgroup of $\Gamma(7,2,5)$.

Remark 3.1. All these maps can be taken with congruent pentagons, and from Theorem 2.2 we obtain configurations of points and circles on the surfaces, in which the circles are isometric in terms of a quotient of the hyperbolic distance.

Figures 3, 4 and 5 show examples of these three distinct geometric realisations of the combinatorial pentagonal geometry of order $(7,7)$ in terms of points and circles, represented in the Poincaré disk. In the case of the realisation coming from the map with automorphism group $C_{7}$, the group action divides the vertex set of the map into seven orbits, each of length seven and one additional fixed point. In Figure 5 the map and the configuration is represented so that the automorphism of order seven is visible as a rotation around the fixed point. It is much harder to visualize the automorphisms of the geometric realisation with automorphism group $C_{5}$. The group action does not fix any vertex, edge nor face of the map.

Proposition 3.2. The dianalytic surfaces of Euler characteristic 55 that admit geometric realisations of the $(7,7)$ pentagonal geometry as a point-circle configuration with different automorphism groups are different.

Proof. Consider the non-orientable Riemann surfaces $S_{i}=\mathbb{H} / G_{i}$ admitting the maps representing the Hoffman-Singleton graph, where $G_{i}$ are torsion-free non-normal subgroups of $\Gamma(7,2,5)$. Note that $\Gamma(7,2,5)$ is a non-arithmetic triangle group [27], and that it is maximal with respect to inclusion [25]. By Theorem 1 in [11], two groups $G$ and $G^{\prime}$, contained 


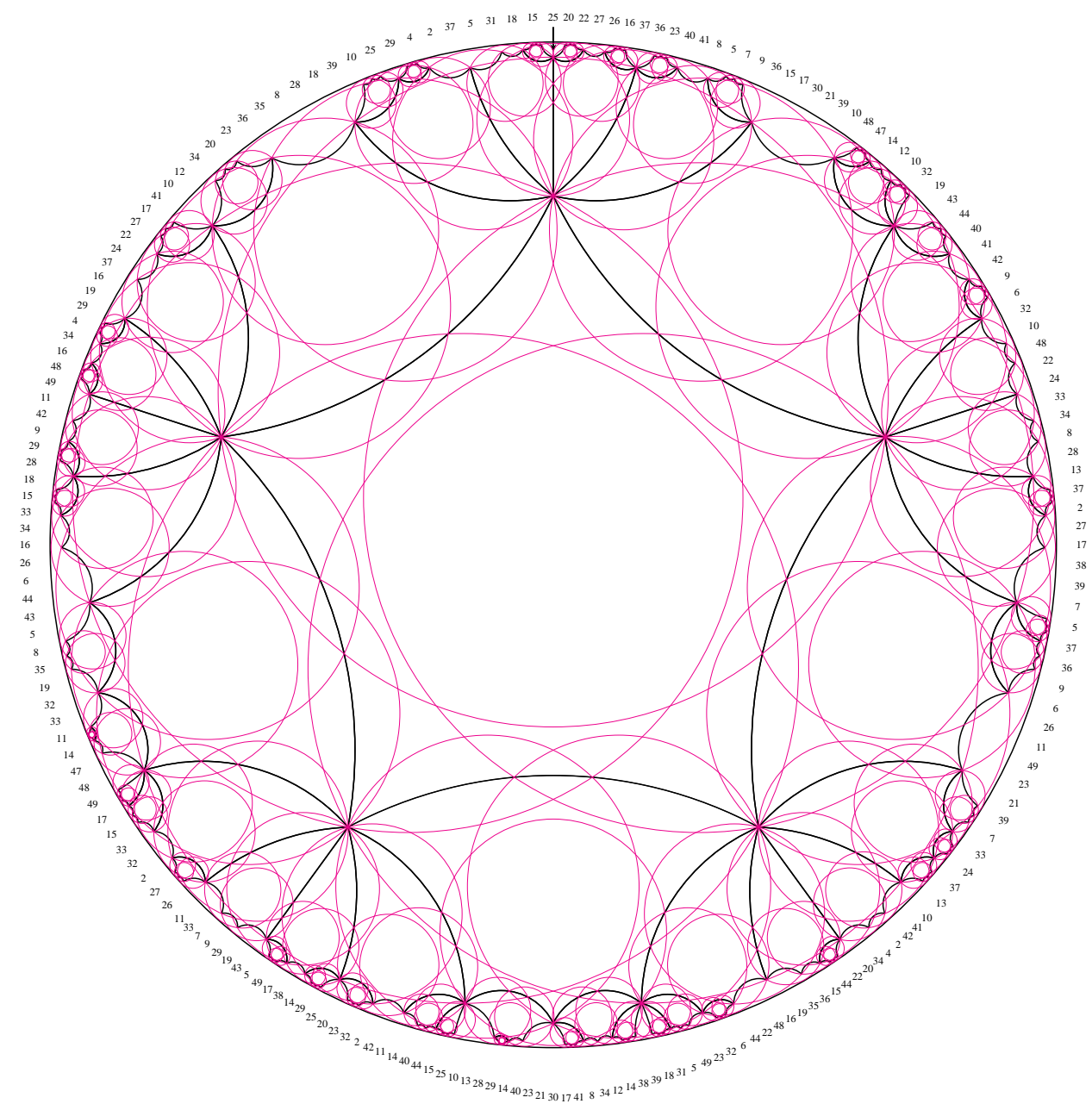

Figure 3: A drawing in a non-orientable surface of genus 57 (identification along the border according to the labelling of the points). The edges colored magenta give the pentagonal geometry of order $(7,7)$. The edges colored black give the pentagonal map of the HoffmanSingleton graph. The automorphism group of this realisation is the trivial group. 


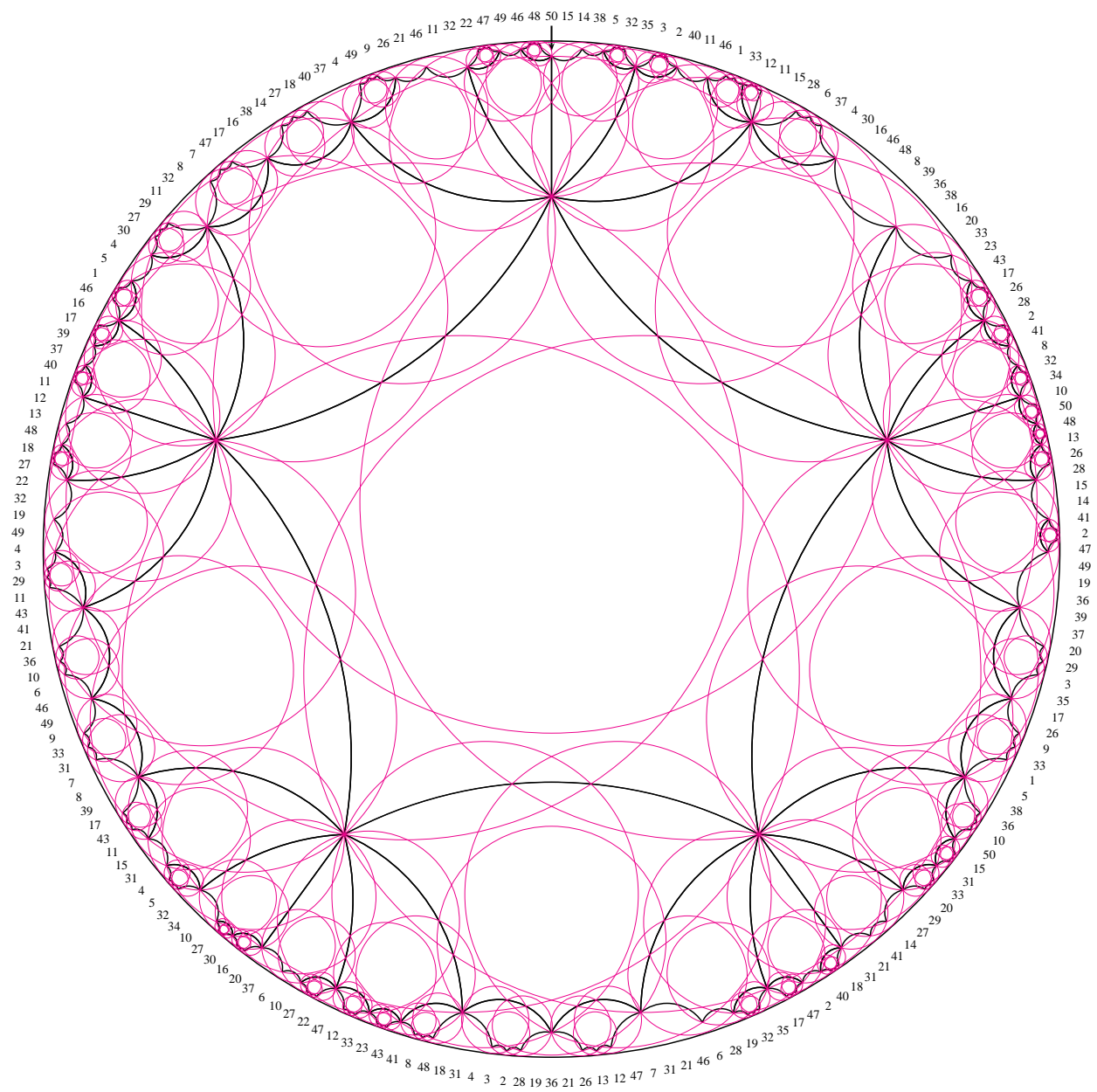

Figure 4: A drawing in a non-orientable surface of genus 57 (identification along the border according to the labelling of the points). The edges colored magenta give the pentagonal geometry of order $(7,7)$. The edges colored black give the pentagonal map of the HoffmanSingleton graph. The automorphism group of this realisation is the cyclic group of order five. 


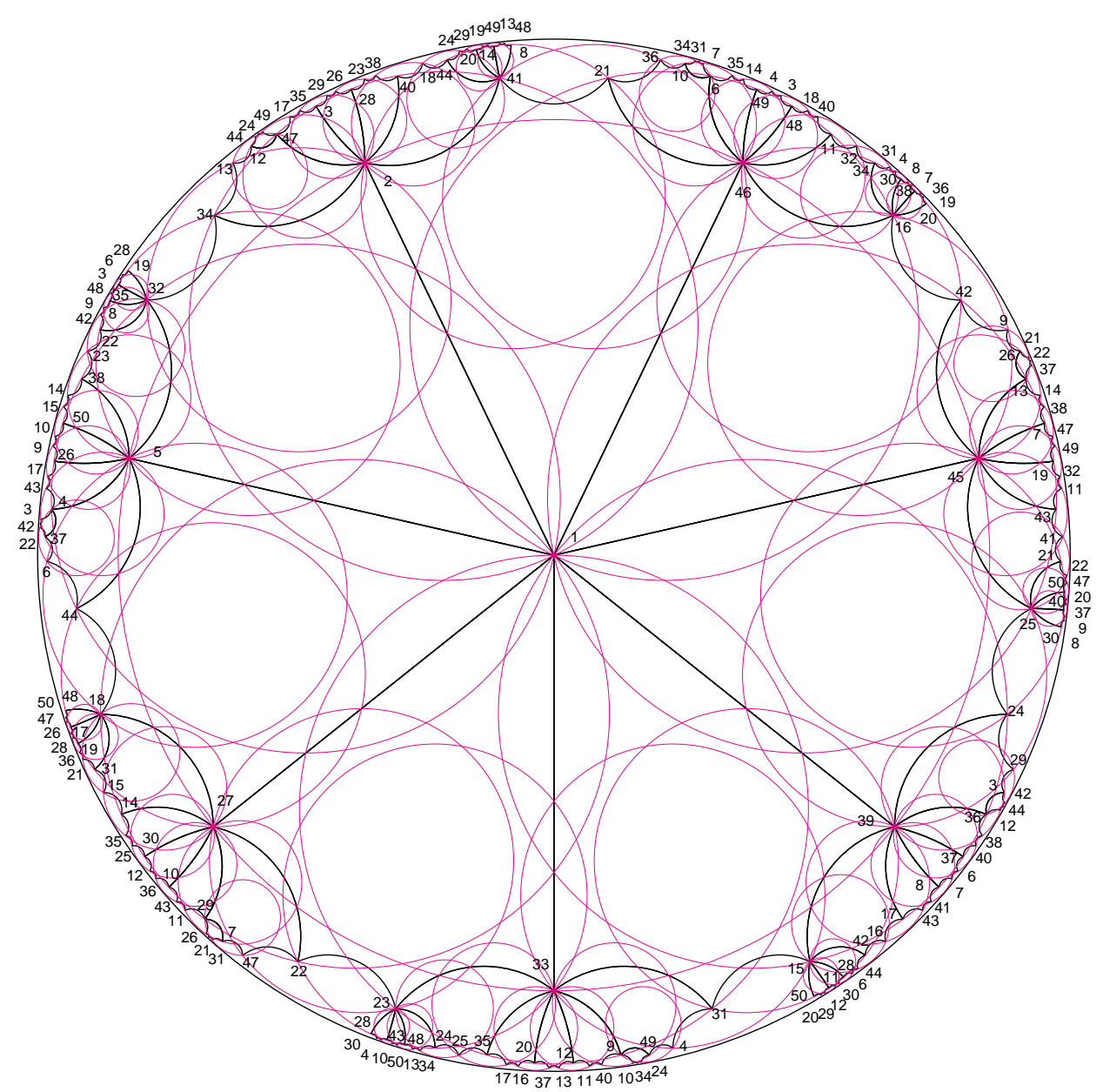

Figure 5: A drawing in a non-orientable surface of genus 57 (identification along the border according to the labelling of the points). The edges colored magenta give the pentagonal geometry of order $(7,7)$. The edges colored black give the pentagonal map of the HoffmanSingleton graph. The automorphism group of this realisation is the cyclic group of order 7. 
in a non-arithmetic Fuchsian triangle group $\Gamma(p, r, q)$, are the uniformizing groups of two dianalytically equivalent surfaces if and only if they are conjugate in a maximal Fuchsian triangle group extending $\Gamma(p, r, q)$, and so the result follows.

Note that Coxeter realised Desargues configuration by embedding its Menger graph (collinearity graph) as a map on a surface of Euler characteristic -5. The edges of the Menger graph nicely line up along the geoedesics. A similar geometric realisation of the $(7,7)$ pentagonal geometry is impossible. Indeed, in this case there are 7 points on each line and 7 lines through each point, so that at each vertex in the Menger graph there are 49 edges. If a combinatorial line with 7 points is represented by a geodesic on this surface, then all the edges between the vertices representing these points in the embedded Menger graph must be on this geodesic. Therefore the geometric representations of these edges would partially overlap, but this is never the case in a map.

\subsubsection{The (7,7)-pentagonal geometry as a point-hypersphere configuration in 24 di- mensional Euclidean space}

The Leech lattice is a Euclidean unimodular lattice in 24 dimensions with extraordinary properties. It can be constructed from the Golay code and provides the optimal kissing configuration of unit balls (hyperspheres) in 24 dimensions and the densest lattice ball packing in $\mathbb{E}^{24}$. Each unit ball touches 196560 other unit balls. The Leech lattice was found in 1967 by Leech [19].

There is a construction of the Higman-Sims graph in the Leech lattice [7]. Start with three lattice points forming the vertices of a triangle with sides of length $2, \sqrt{6}$ and $\sqrt{6}$. The number of lattice points at distance 2 from at least one of the vertices of the triangle is exactly 100 . Construct a graph with these 100 points as vertices and with an edge between two points whenever the distance between them is $\sqrt{6}$. Then this graph is the Higman-Sims graph with automorphism group the Higman-Sims sporadic simple group HS. It is wellknown that the vertex set of the Higman-Sims graph can be partitioned into two copies of the Hoffman-Singleton graph. The automorphism groups of these two copies of the Hoffman-Singleton graph in the Leech lattice are two conjugate subgroups of HS, each isomorphic to $P S U(3,5) \rtimes C_{2}$, the automorphism group of the combinatorial HoffmanSingleton graph.

Note that the edges in one of these embeddings of the Hoffman-Singleton graph all have length $\sqrt{6}$. Hence there is a hypersphere centered at each vertex of radius $\sqrt{6}$, such that the graph vertices contained in each hypersphere are exactly those that are adjacent to the vertex in the center. Indeed, this is (more or less) Theorem 2.2 for the Euclidean plane generalized to higher dimensions. The result is a geometric realisation of the $(7,7)$-pentagonal geometry as a point-hypersphere configuration in 24 dimensions. The automorphism group of this embedding of the Hoffman-Singleton graph is $P S U(3,5) \rtimes C_{2}$. Since this is the automorphism group of the combinatorial object, this is the largest possible.

The embedding of the Hoffman-Singleton graph in the Leech lattice is not unit-distance, but it is isometric, as required by the construction in Theorem 2.2. It was proved by Maehara and Rödl that any graph of maximum valency $d$ can be embedded as a unit-distance graph in $\mathbb{E}^{2 d}$ [21], however this does not say anything about the symmetry group of the embedding. 


\subsection{The pentagonal geometries of order $(k, k+1)$}

Since all pentagonal geometries of order $(k, k+1)$ can be constructed from pentagonal geometries of order $(k+1, k+1)$ through the removal of one point and its opposite line, there are at most three (connected) pentagonal geometries of order $(k, k+1)$, with $k=2,6$ and maybe 56. The automorphism group of these combinatorial pentagonal geometries is the point-stabilizer of the automorphism group of the corresponding combinatorial pentagonal geometry of order $(k+1, k+1)$. The pentagonal geometry of order $(2,3)$ is constructed from the Desargues configuration and has automorphism group $S_{3} \times C_{2}$. The pentagonal geometry of order $(6,7)$ has automorphism group $S_{7}$.

As a consequence of the construction of pentagonal geometries of order $(k, k+1)$ from those of order $(k+1, k+1)$, any geometric realisation of a $(k+1, k+1)$ pentagonal geometry gives rise to a geometric realisation of the corresponding pentagonal geometry of order $(k, k+1)$, by simply removing from the realisation a point and the geometric realisation of its opposite combinatorial line. In the case of point-circle (point-hypersphere) realisations, the geometric realisation of a combinatorial line is a circle (hypersphere). Therefore any geometric realisation, in terms of points and circles (or hyperspheres), of a pentagonal geometry of order $(k+1, k+1)$, described previously in this article, gives rise to a geometric realisation in terms of points and circles (or hyperspheres) of the corresponding pentagonal geometry of order $(k, k+1)$.

The automorphism group of the geometric realisation of the pentagonal geometry of order $(k, k+1)$ is the intersection of the point-stabilizer of the combinatorial pentagonal geometry of order $(k+1, k+1)$ and the automorphism group of its corresponding geometric realisation.

\section{References}

[1] S. Ball, J. Bamberg, A. Devillers and K. Stokes, An alternative way to generalize the pentagon, J. Combin. Des. 21 (2013), 163-179, doi:10.1002/jcd.21325.

[2] C. T. Benson and N. E. Losey, On a graph of Hoffman and Singleton, J. Combinatorial Theory Ser. B 11 (1971), 67-79.

[3] A. E. Brouwer and J. H. van Lint, Strongly regular graphs and partial geometries, in: Enumeration and design (Waterloo, Ont., 1982), Academic Press, Toronto, ON, 1984 pp. 85-122.

[4] R. P. Bryant and D. Singerman, Foundations of the theory of maps on surfaces with boundary, Quart. J. Math. Oxford Ser. (2) 36 (1985), 17-41, doi:10.1093/qmath/36.1.17.

[5] M. Conder and B. Everitt, Regular maps on non-orientable surfaces, Geom. Dedicata 56 (1995), 209-219, doi:10.1007/BF01267644.

[6] M. Conder and K. Stokes, Minimum genus embeddings of the Hoffman-Singleton graph.

[7] J. H. Conway and N. J. A. Sloane, Sphere packings, lattices and groups, volume 290 of Grundlehren der Mathematischen Wissenschaften [Fundamental Principles of Mathematical Sciences], Springer-Verlag, New York, 3rd edition, 1999, doi:10.1007/978-1-4757-6568-7.

[8] H. S. M. Coxeter, Desargues configurations and their collineation groups, Math. Proc. Cambridge Philos. Soc. 78 (1975), 227-246.

[9] G. Gévay, Symmetric configurations and the different levels of their symmetry, Symmetry Cult. Sci 20 (2009), 309-329.

[10] G. Gévay and T. Pisanski, Kronecker covers, $V$-construction, unit-distance graphs and isometric point-circle configurations, Ars Math. Contemp. 7 (2014), 317-336. 
[11] E. Girondo, D. Torres-Teigell and J. Wolfart, Shimura curves with many uniform dessins, Math. Z. 271 (2012), 757-779, doi:10.1007/s00209-011-0889-4.

[12] G. González-Diez, Variations on Belyi’s theorem, Q. J. Math. 57 (2006), 339-354, doi:10. 1093/qmath/hai021.

[13] B. Grünbaum, Configurations of points and lines, volume 103 of Graduate Studies in Mathematics, American Mathematical Society, Providence, RI, 2009, doi:10.1090/gsm/103.

[14] W. H. Haemers, Eigenvalue techniques in design and graph theory, volume 121 of Mathematical Centre Tracts, Mathematisch Centrum, Amsterdam, 1980, dissertation, Technische Hogeschool Eindhoven, Eindhoven, 1979.

[15] P. R. Hafner, The Hoffman-Singleton graph and its automorphisms, J. Algebraic Combin. 18 (2003), 7-12, doi:10.1023/A:1025136524481.

[16] D. Hilbert and S. Cohn-Vossen, Geometry and the imagination, Chelsea Publishing Company, New York, N. Y., 1952, translated by P. Neményi.

[17] A. J. Hoffman and R. R. Singleton, On Moore graphs with diameters 2 and 3, IBM J. Res. Develop. 4 (1960), 497-504.

[18] G. A. Jones and D. Singerman, Theory of maps on orientable surfaces, Proc. London Math. Soc. (3) 37 (1978), 273-307.

[19] J. Leech, Notes on sphere packings, Canad. J. Math. 19 (1967), 251-267.

[20] C. Lefèvre-Percsy, N. Percsy and D. Leemans, New geometries for finite groups and polytopes, Bull. Belg. Math. Soc. Simon Stevin 7 (2000), 583-610.

[21] H. Maehara and V. Rödl, On the dimension to represent a graph by a unit distance graph, Graphs Combin. 6 (1990), 365-367, doi:10.1007/BF01787703.

[22] T. Pisanski and B. Servatius, Configurations from a graphical viewpoint, Birkhäuser Advanced Texts: Basler Lehrbücher. [Birkhäuser Advanced Texts: Basel Textbooks], Birkhäuser/Springer, New York, 2013, doi:10.1007/978-0-8176-8364-1.

[23] G. N. Robertson, Graphs minimal under girth, valency and connectivity constraints, ProQuest LLC, Ann Arbor, MI, 1969, thesis (Ph.D.)-University of Waterloo (Canada).

[24] I. Shimada, The graphs of Hoffman-Singleton, Higman-Sims and McLaughlin, and the Hermitian curve of degree 6 in characteristic 5, Australas. J. Combin. 59 (2014), 161-181.

[25] D. Singerman, Finitely maximal Fuchsian groups, J. London Math. Soc. (2) 6 (1972), 29-38.

[26] D. Singerman and R. I. Syddall, The Riemann surface of a uniform dessin, Beiträge Algebra Geom. 44 (2003), 413-430.

[27] K. Takeuchi, Arithmetic triangle groups, J. Math. Soc. Japan 29 (1977), 91-106.

[28] K. G. C. von Staudt, Geometrie der lage, Bauer und Raspe, 1847. 